\title{
Cold-Stress Response of Probiotic Lactobacillus plantarum K25 by iTRAQ Proteomic Analysis ${ }^{\mathbb{S}}$
}

\author{
Shaoli Liu ${ }^{1,2}$, Yimiao $\mathrm{Ma}^{1,2}$, Yi Zheng ${ }^{1,2}$, Wen Zhao ${ }^{1,2}$, Xiao Zhao ${ }^{1,2}$, Tianqi Luo ${ }^{1,2}$, Jian Zhang ${ }^{1,2}$, and Zhennai \\ Yang $^{1,2 *}$ \\ ${ }^{1}$ Beijing Advanced Innovation Center for Food Nutrition and Human Health, Beijing Technology and Business University Beijing, P.R. China \\ ${ }^{2}$ Engineering and Technology Research Center of Food Additives, Beijing Technology and Business University, Beijing, 100048, P.R. China
}

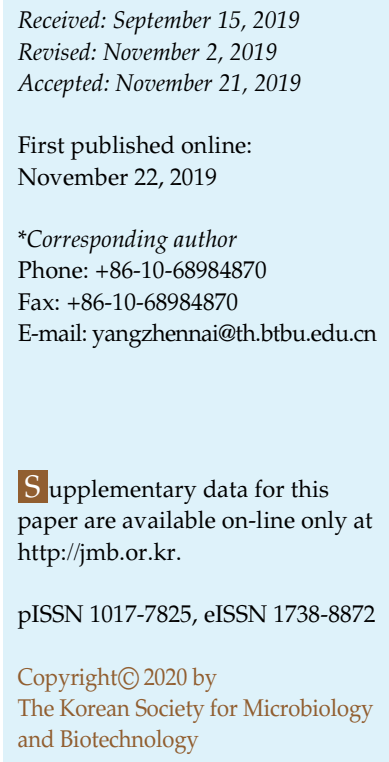

To understand the molecular mechanism involved in the survivability of cold-tolerant lactic acid bacteria was of great significance in food processing, since these bacteria play a key role in a variety of low-temperature fermented foods. In this study, the cold-stress response of probiotic Lactobacillus plantarum K25 isolated from Tibetan kefir grains was analyzed by iTRAQ proteomic method. By comparing differentially expressed (DE) protein profiles of the strain incubated at $10^{\circ} \mathrm{C}$ and $37^{\circ} \mathrm{C}, 506 \mathrm{DE}$ proteins were identified. The DE proteins involved in carbohydrate, amino acid and fatty acid biosynthesis and metabolism were significantly down-regulated, leading to a specific energy conservation survival mode. The DE proteins related to DNA repair, transcription and translation were up-regulated, implicating change of gene expression and more protein biosynthesis needed in response to cold stress. In addition, two-component system, quorum sensing and ABC (ATP-binding cassette) transporters also participated in cell cold-adaptation process. These findings provide novel insight into the cold-resistance mechanism in L. plantarum with potential application in low temperature fermented or preserved foods.

Keywords: Proteomics, iTRAQ, Lactobacillus plantarum K25, cold tolerance

\section{Introduction}

Lactic acid bacteria (LAB) play important roles in food fermentation in providing positive attributes to food sensory properties, quality and safety [1]. During fermentation and storage periods, these bacteria may encounter different stress conditions, e.g. low temperature, low $\mathrm{pH}$, high ethanol concentration, high osmotic pressure and high salt stress, etc. [2, 3]. Traditional food fermentation is often executed under relative coldness conditions $\left(0-10^{\circ} \mathrm{C}\right)$, e.g. Korean kimchi, pickled cabbage, fermented bean curd and cucumber, etc., where there is presence of cold-resistant LAB strains surviving the cold stress conditions. In fermented ice cream, rigorous low temperature aging, freezing and preserving are experienced by LAB and the tolerant strains may survive during the production processes [2]. Although numerous LAB have been identified and utilized from traditional fermented products, the cold- resistant $\mathrm{LAB}$ have long been used without being fully understood.

It was known that cold-resistant LAB could adapt to a cold environment, maintaining cell viability and producing more lactic acid to enhance product flavor and health properties [3]. However, cold stress would cause changes in membrane fluidity (influencing nutrient transport), lowering of enzyme activity (influencing cellular metabolism) and RNA structure stability (influencing translation process), all of which further influence the bacteria viability [4]. During the past few decades, there have been numerous studies exploring cold resistance mechanisms in pathogenic bacteria such as Listeria monocytogenes, Cronobacter turicensis, Staphylococcus aureus, Escherichia coli and environmental microorganisms such as Cryptococcus humicola and Desulfovibrio vulgaris, as they could survive during low temperature preservation and pose a great threat to food safety, whereas only a few studies have focused on LAB [5-9]. 
The main opinions about cold resistance mechanisms have mainly been related to changes in cell morphology, decreased membrane fluidity and increased integrity, modified carbohydrate and amino acid biosynthesis metabolism, and increased RNA degradation rate to counterbalance the RNA stabilization [10]. Numerous chaperones and transcription regulators also play important roles since the cold shock protein family has been identified as a main factor in cold adaptation [11]. Due to their wide potential application, further investigation into the cold-adaptation mechanisms of LAB may lead to discovery of new agents or methods that might also facilitate the adaptation and growth of LAB at low temperature.

Lactobacillus plantarum is commonly found in various environment niches, including those under extreme conditions such as low $\mathrm{pH}$, high osmotic pressure and low temperature, etc., where there is presence of other tolerant strains adapting to the stress conditions. Previously, $L$. plantarum K25 isolated from low temperature preserved Tibetan Kefir grains was characterized as a probiotic strain [12]. In this study, the growth characteristics of L. plantarum K25 at low temperature were investigated, and the molecular mechanism involved in the cold adaptation of the strain was studied by iTRAQ proteomic analysis by comparing the protein profiles expressed by the strain at $10^{\circ} \mathrm{C}$ and $37^{\circ} \mathrm{C}$, respectively. The temperature at $10^{\circ} \mathrm{C}$ was used to mimic the cold fermentation and storage condition. The protein samples were prepared from the strain at its optimal growth in this study. The present study may provide further insight into the cold-adaptation strategy employed by L. plantarum under cold fermentation and storage conditions.

\section{Materials and Methods}

\section{Bacterial Strain and Growth Conditions}

L. plantarum K25 was provided by Jilin Academy of Agricultural Science of China. It was isolated from Tibetan Kefir with antioxidant and gut microbiota regulating properties [13, 14]. Lactobacillus rhamnosus GG (LGG, ATCC: 53103) obtained from the LAB collection in the authors' lab were used for comparison of growth at low temperature. For studies on cold response, these strains were grown in MRS medium at $10^{\circ} \mathrm{C}$ and $37^{\circ} \mathrm{C}$, respectively. At 0 , $2,4,8,10,12,16,24,36,48,60,72,84,96 \mathrm{~h}$ after inoculating, the medium was determined for absorbance of $\mathrm{OD}_{600 \mathrm{~nm}}$ by spectrophotometer. At the corresponding time point, $\mathrm{pH}$ was detected respectively. Each growth experiment was repeated three times.

\section{Protein Extraction and Quantification}

Bacterial cell samples were prepared by centrifugation of the culture samples obtained from the optimal growth (about the end of the logarithmic growth phase) of L.plantarum $\mathrm{K} 25$ at $10^{\circ} \mathrm{C}$ and $37^{\circ} \mathrm{C}$, respectively, and a sample of the latter was used as control. Three independent replicates were collected for each sample. The cell pellets obtained from centrifugation of the bacterial culture were resuspended and washed 3 times with PBS (phosphatebuffered saline). The lysis solution (1 $\mathrm{ml}$ of lysis buffer contained $1 \mu \mathrm{l}$ protease inhibitor, $10 \mu \mathrm{l}$ of $1 \mathrm{M}$ DTT and $10 \mu \mathrm{l}$ of $100 \mathrm{mM}$ PMSF) was added to one-fifth of the original volume of the bacterial cultures, and the mixture was incubated at $4^{\circ} \mathrm{C}$ for $10 \mathrm{~min}$. The cells were further lysed by ultra-sonication and centrifuged at $14,000 \times g$ for $15 \mathrm{~min}$ at $4^{\circ} \mathrm{C}$. Supernatant was collected and the protein concentration was quantified using the Bradford method.

\section{Protein Digestion and iTRAQ Labeling}

Approximately $200 \mu \mathrm{g}$ of protein was mixed with $4 \mu \mathrm{L}$ DTT and incubated at $37^{\circ} \mathrm{C}$ for $1 \mathrm{~h}$. Then the mixture was added with $20 \mu \mathrm{l}$ iodoacetamide and incubated for $0.5-1 \mathrm{~h}$ at room temperature. The alkylated protein was collected by centrifugation at 12,000 $\times g$ for $20 \mathrm{~min}$. Subsequently, the protein was dissolved with $120 \mu \mathrm{l}$ solution $(8 \mathrm{M}$ urea and $100 \mathrm{ml}$ Tris- $\mathrm{HCl})$ and centrifuged at $12,000 \times g$ for $10 \mathrm{~min}$, and this procedure was repeated twice. The supernatant was added with $100 \mu \mathrm{l}$ TEAB buffer solution and centrifuged at $12,000 \times g$ for $10 \mathrm{~min}$. After washing three times, the pellet was digested with $4 \mu \mathrm{l}$ trypsin solution at $37^{\circ} \mathrm{C}$ overnight. The digested samples were then centrifuged at $12,000 \times g$ for $10 \mathrm{~min}$, and the supernatant was discarded. iTRAQ labeling was done by using an iTRAQ Reagent 8Plex Multiplex Kit (4381663, SigmaAldrich, USA) according to the manufacturer's instructions. iTRAQ reagent was used to coordinately label the peptides from the control samples $\left(37^{\circ} \mathrm{C}\right)$ and the experimental samples $\left(10^{\circ} \mathrm{C}\right)$. The labeled peptides were incubated at room temperature for $1 \mathrm{~h}$ and reaction was stopped by adding 50-100 $\mu$ l of ultrapure water. Then, $1 \mu \mathrm{l}$ of sample was removed from each group and mixed to confirm that the samples had been labeled successfully. Finally, the labeled peptides were pooled and lyophilized.

\section{LC-MS/MS Analysis and Database Search}

The peptide sample $(100 \mu \mathrm{g})$ was dissolved with $500 \mu \mathrm{NH}_{4} \mathrm{HCO}_{3}$, and eluted with a Strata-X C18 column using $1 \mathrm{ml} \mathrm{0.1 \%} \mathrm{FA} \mathrm{and} \mathrm{8 \%}$ acetonitrile, and $1 \mathrm{ml}$ eluate liquid was collected and lyophilized. The dried sample was re-dissolved in $20 \mu \mathrm{l} 0.5 \mathrm{M}$ TEAB and divided into 12 fractions. The analytical separation was performed using an Eksigent Chromxp Trap Column $(350 \mu \mathrm{m} \times 0.5 \mathrm{~nm}, 120 \mathrm{~A}$, $3 \mu \mathrm{m}$ ) and a pulled quartz tip as the emitter (New Objectives, USA). LC was performed with a flow rate of $0.3 \mathrm{ml} / \mathrm{min}$ using the following elution program: 0-65min: $95 \%$ buffer A $(0.1 \%$ formic acid $+5 \%$ acetonitrile $)+5 \%$ buffer B $(0.1 \%$ formic acid $+95 \%$ acetonitrile); $65-70 \mathrm{~min}$ : 70\% buffer $\mathrm{A}+30 \%$ buffer $\mathrm{B} ; 70-80 \mathrm{~min}$ : $50 \%$ buffer $A+50 \%$ buffer $B ; 80-85$ min: $20 \%$ buffer $A+80 \%$ buffer B; 85-90 min: $95 \%$ buffer $A+5 \%$ buffer B. 


\section{Data Analysis}

The original data files gathered by MS in wild format were processed with Protein Pilot Software V4.5 (AB Sciex, USA) against the L. plantarum K25 protein database using the Paragon algorithm. For protein identification, a mass tolerance of $0.05 \mathrm{Da}$ was permitted for intact peptide masses and 0.1 Da for fragmented masses, with allowance for one missed cleavage upon trypsin digest. Several parameters in Mascot were set for peptide searching, including iTRAQ quantification (N-term, K), cysteine modified with iodoacetamide, and trypsin digestion. A protein containing at least two unique peptides was required for quantitation. The quantitation protein ratios were weighted and normalized by the median ratio in Mascot. Protein with at least 1.5 -fold change between two samples and a $p$ value $<0.05$ was considered as one significantly differential species. COG (Cluster of Orthologous Groups of proteins) analysis was conducted according to the NCBI database (http://www.ncbi.nlm.nih.gov/ COG/).

\section{RNA Extraction and Quantitative Transcriptional Analysis}

All gene manipulation was based on the genome of L. plantarum K25 in NCBI. RNA was extracted using a Universal RNA Extraction Kit (TianGen, China) according to the instructions. RNA yield and quality were evaluated with a UV spectrometer Q5000 (Quwell). Then, approximately $4 \mathrm{ul}$ of RNA was reverse transcribed with a Tastline Cell DNA Kit (TianGen). Quantitative real-time PCR (qRT-PCR) was performed using the Real Master Mix (TianGen) with a final volume of $25 \mathrm{ul}$ in each reaction system, which included $12.5 \mathrm{ul}$ Mix containing SYBR Green 1, dNTPs, AmpliTaq Gold Fast DNA polymerase LD, 10x buffer, and $1 \mathrm{ul} \mathrm{cDNA}, 0.5 \mathrm{ul}$ of each primer and $10.5 \mathrm{ul}$ of $\mathrm{ddH}_{2} \mathrm{O}$, the target genes involved in the genome sequence of L. plantarum K25 and primers (designed by Primer Primer 5.0). The amplification procedure included: denaturation at $94^{\circ} \mathrm{C}$ for $2 \mathrm{~min} ; 35$ cycles of $94^{\circ} \mathrm{C}$ for $30 \mathrm{sec} ; 53^{\circ} \mathrm{C}$ for $30 \mathrm{sec} ; 72^{\circ} \mathrm{C}$ for $30 \mathrm{sec}$, and $72^{\circ} \mathrm{C}$ for

A

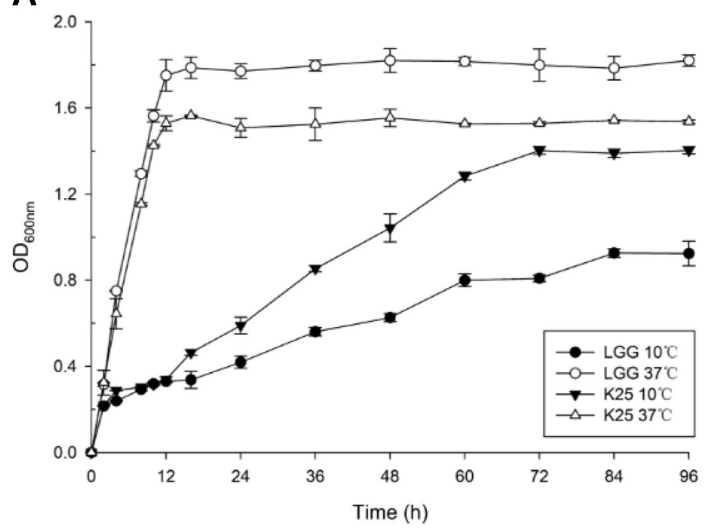

10 min. At the end of PCR cycles, melting curve analyses were performed using the LightCycle Nano qRT-PCR system. All the samples were produced and run in triplicate, and $16 \mathrm{~S}$ rRNA gene was used as the reference. The primer sequences for the representative proteins highly differentially expressed were listed in Table S1.

\section{Results}

\section{Growth Behavior under Low Temperature Condition}

Rather slow growth of L. plantarum K25 was observed at $10^{\circ} \mathrm{C}$ in comparison with its relatively fast growth at $37^{\circ} \mathrm{C}$ as shown by the significantly prolonged and delayed logarithmic growth phase at the low temperature (Fig. 1A). It took about $72 \mathrm{~h}$ for strain $\mathrm{K} 25$ to reach stationary growth at $10^{\circ} \mathrm{C}$, and took about $14 \mathrm{~h}$ at $37^{\circ} \mathrm{C}$. Similarly, LGG also grew more slowly at $10^{\circ} \mathrm{C}$ than at $37^{\circ} \mathrm{C}$. However, strain $\mathrm{K} 25\left(\mathrm{OD}_{600 \mathrm{~nm}} 1.4159\right.$ at $\left.72 \mathrm{~h}\right)$ showed better growth than strain LGG $\left(\mathrm{OD}_{600 \mathrm{~nm}} 0.8497\right.$ at $\left.72 \mathrm{~h}\right)$ at the low temperature. The change of $\mathrm{pH}$ value correlated well to that of $\mathrm{OD}_{600}$ at the corresponding temperature of growth with the $\mathrm{pH}$ values of 3.97 and 4.25 at $72 \mathrm{~h}$ at $10^{\circ} \mathrm{C}$ for strains $\mathrm{K} 25$ and LGG, respectively (Fig. 1B). The results indicated that both strains of K25 and LGG might develop a mechanism to adapt to the long-term low temperature condition, though they exhibited different degrees of tolerance to the low temperature. In the following study, L. plantarum K25 was selected for proteomic analysis of its response to low temperature $\left(10^{\circ} \mathrm{C}\right)$ stress in comparison with that to its normal growth condition $\left(37^{\circ} \mathrm{C}\right)$.

\section{Primary Data Analysis and Protein Identification}

It is significant to explore the cold-adaptation mechanism

\section{B}

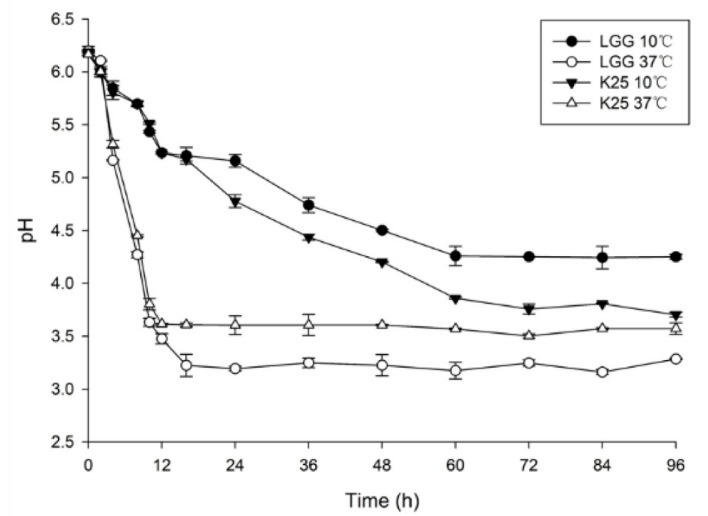

Fig. 1. Growth behavior of L. plantarum $\mathrm{K} 25$ and L. rhamnusus $\mathrm{GG}$ at $10^{\circ} \mathrm{C}$ and $37^{\circ} \mathrm{C}$ in the MRS medium as monitored by measurement of $\mathrm{OD}_{600 \mathrm{~nm}}(\mathbf{A})$ and $\mathrm{pH}(\mathbf{B})$. 
A

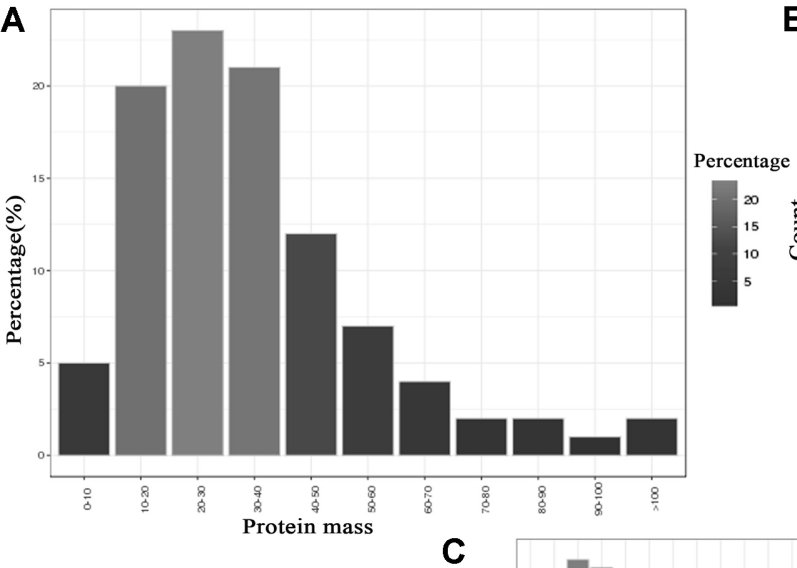

B

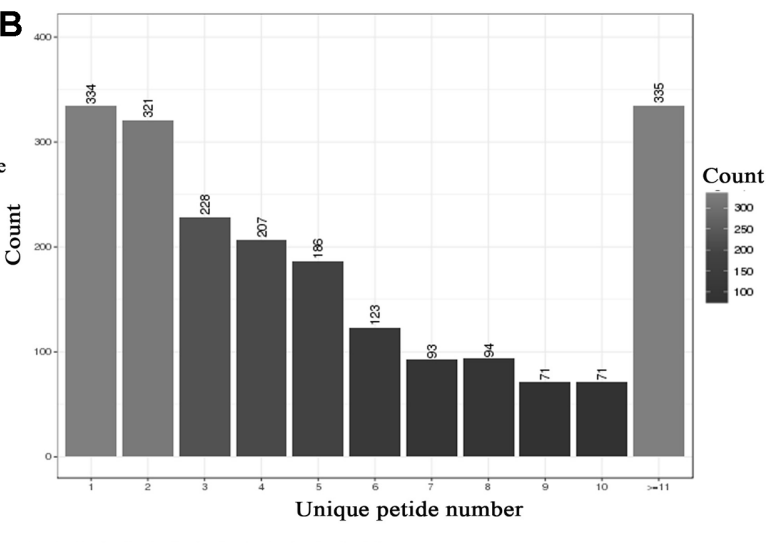

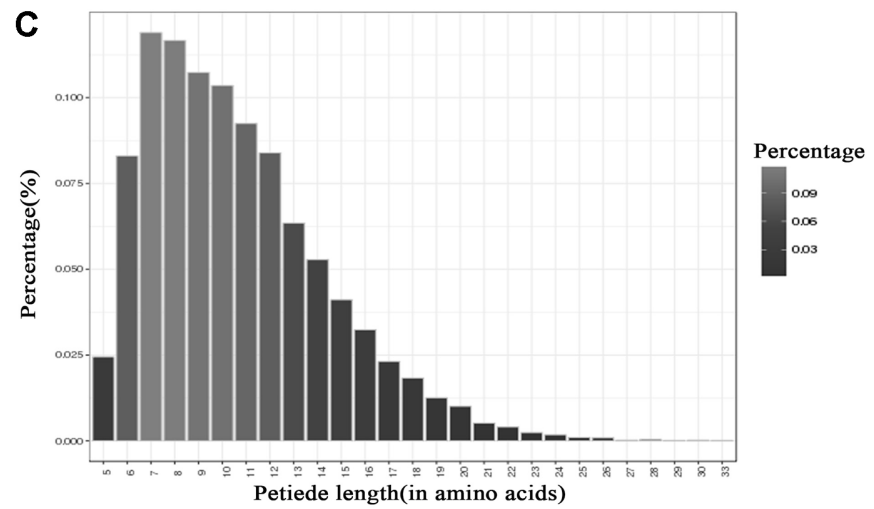

Fig. 2. Identification and analysis of the L. plantarum K25 proteome.

(A) Identified proteins were based on their protein mass. (B) Numbers of unique peptides that match to proteins. (C) Percentages of peptide length (in amino acids).

for LAB involved in food processing at low temperature. Using iTRAQ-based technology in our study enabled us to further extend the search scope for differentially expressed proteins at low temperature when compared with traditional 2-DE proteome studies. Among total of 227,107 spectra obtained, 49,106 spectra were identified including 12,483 unique peptides and 2,063 identified proteins before grouping with a $1 \%$ false discovery rate (FDR) as the cutoff in two independent experiments. There were $83 \%, 15 \%$ and $2 \%$ of proteins with molecular mass of $0-50 \mathrm{kDa}, 50-100 \mathrm{kDa}$ and $>100 \mathrm{kDa}$, respectively (Fig. 2A). The distribution of peptide numbers is shown in Fig. 2B. These proteins with single peptide, 2-4 peptides, 5-7 peptides, 8-10 peptides and above 11 peptides consisted of 334, 756, 392, 236 and 355 , respectively. A total $83.89 \%$ of proteins contained at least 2 peptides. Unique peptide length was between 5 and 72 (in amino acids) (Fig. 2C).

\section{Analysis and Functional Annotation of Differential Proteins}

A total of 509 common DE proteins were found. The DE proteins and details about their functions were summarized in Table S2. A Gene Ontology (GO) enrichment analysis was performed to classify the differentially expressed proteins according to their biological process, location in cellular component, and molecular function. Functional assignment for each protein was made using the nomenclature developed for clusters of orthologous groups of proteins (COG). In the category of cellular components, the DE proteins were mainly located in cell organelle and membrane; in terms of molecular function, the DE proteins mainly executed catalytic and binding activity; for biological processes, the DE proteins were mainly involved in cellular process, metabolic process, response to stimulus and biological regulation (Fig. 3A).

The KEGG pathway enrichment analysis was conducted to determine the biological pathway to which the DE proteins belonged (Fig. 3B). In the different temperature environments, $29 \mathrm{DE}$ proteins were involved in carbon metabolism, such as pyruvate metabolism (path: ko00620), glycolysis/gluconeogenesis (path: ko00010) and citrate acid 


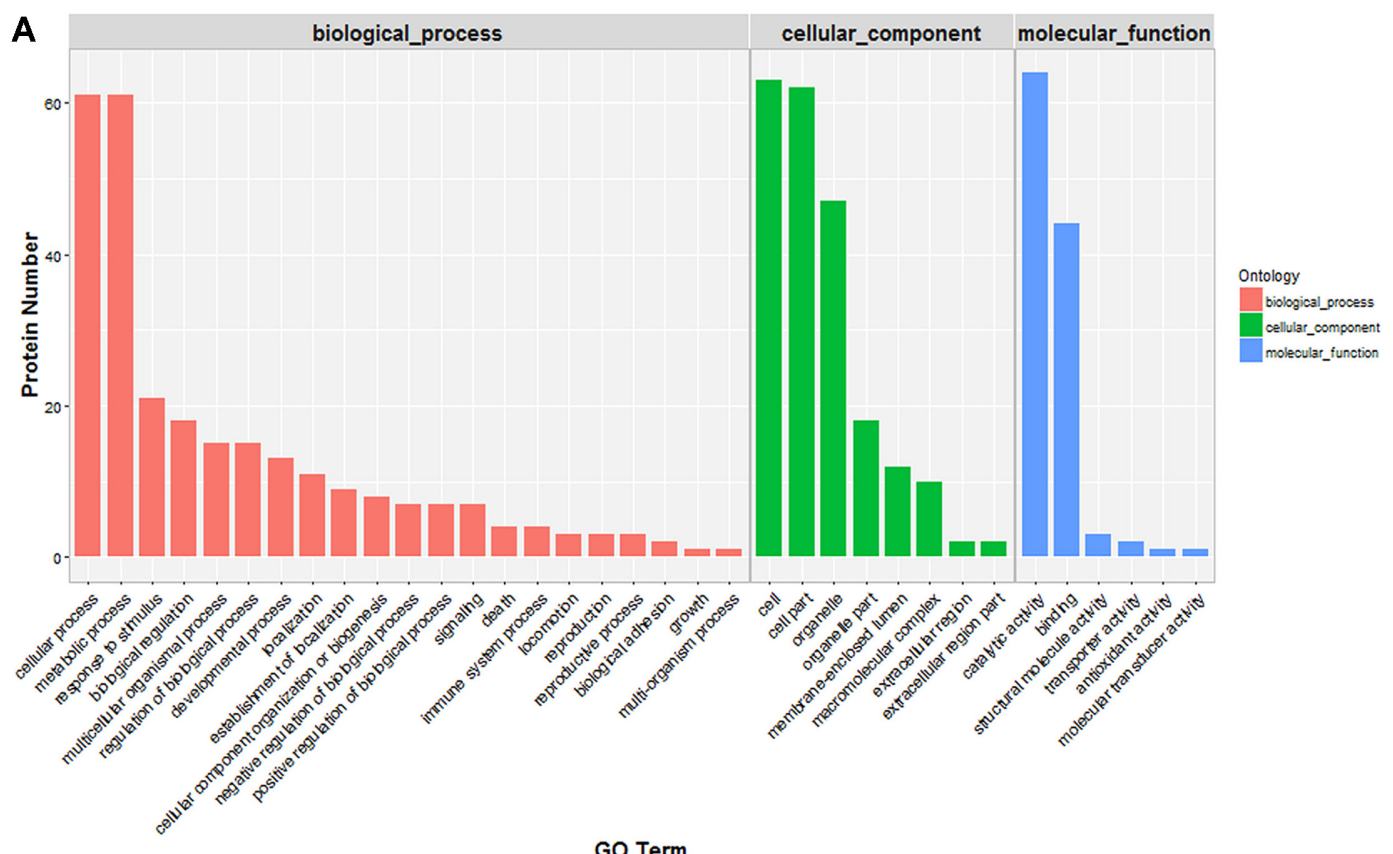

B

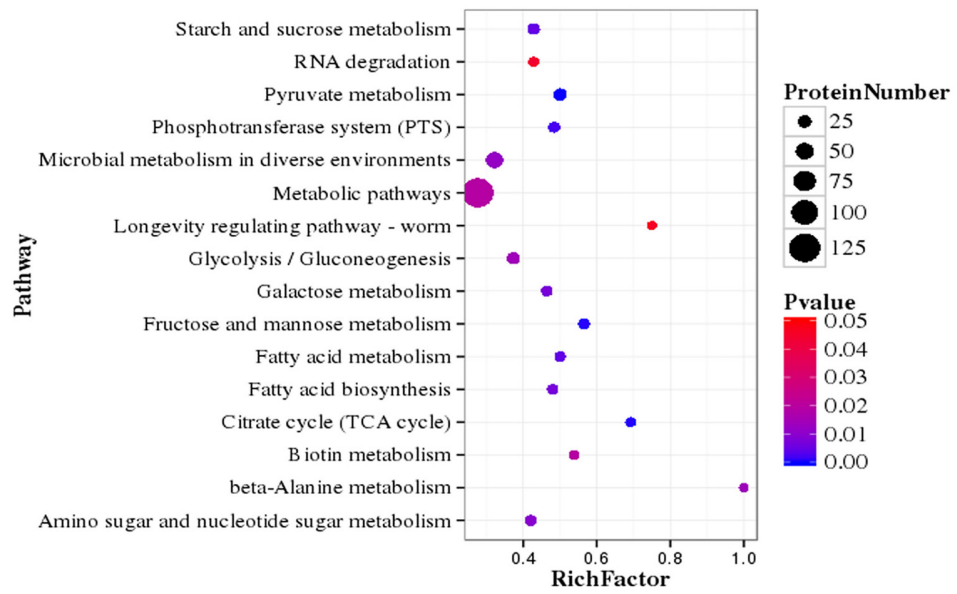

Fig. 3. GO and KEGG annotation of distribution analysis.

(A) Functional categorization of identified proteins according to the Gene Ontology Consortium classification. The vertical scales are protein numbers. Blue bars refer to DE proteins involved in molecular component; Green bars refer to DE proteins in cellular component; Pink bars refer to DE proteins involved in biological process. (B) Pathway classification based on KEGG enrichment analysis of differentially expressed proteins of L. plantarum $\mathrm{K} 25$ in response to cold stress. Rich factor, the ratio of the number of differentially expressed genes to the number of total genes in this pathway. Proteins with at least 1.5 -fold change are shown; adjusted $p \leq 0.05$ for all data selected.

cycle (TCA) (path: ko00020), indicating that carbohydrate metabolism might be the main factor influencing cell survival at $10^{\circ} \mathrm{C}$. In comparison, only $16 \mathrm{DE}$ proteins were related to amino acid metabolism and biosynthesis, including beta-alanine metabolism (path: ko00410), lysine biosynthesis (path: ko00300), and histidine metabolism (path: ko00340). There were $14 \mathrm{DE}$ proteins involved in fatty acid metabolism (path: ko01212) and biosynthesis (path: ko00061). Ribosome and protein metabolism might be the critical biological process for cell viability. There were approximately $55 \mathrm{DE}$ proteins involved in transcription and translation processes, such as RNA degradation (path: ko03018), protein export (path: ko03060), ribosome (path: ko03010). Additionally, proteins involved in peptidoglycan biosynthesis (path: ko00550), two-component system (path: ko02020), ABC transporters (path: ko02010) and phospho- 


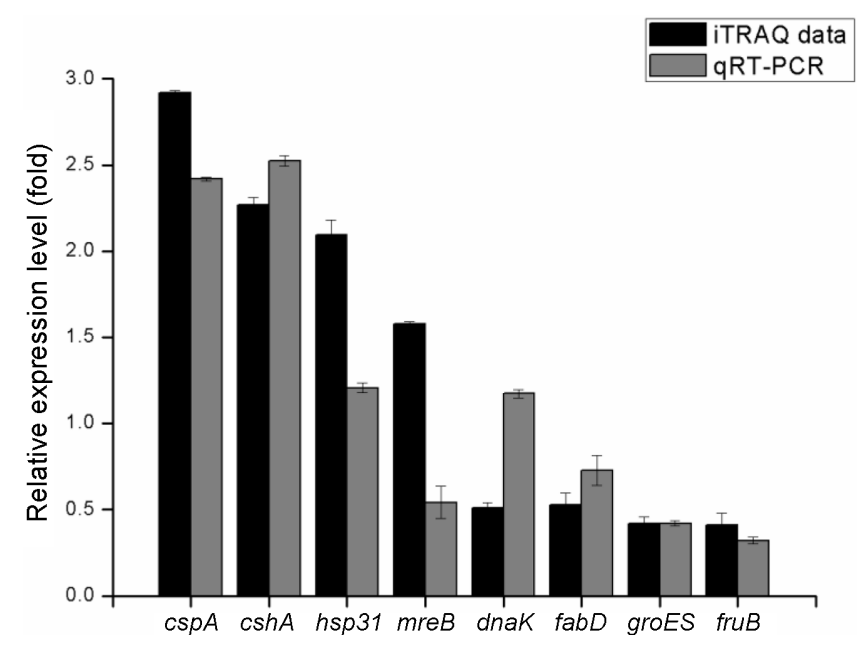

Fig. 4. Comparative analysis of the transcripts (mRNA levels) and protein levels of the representative differentially expressed proteins revealed by qRT-PCR and iTRAQ, respectively, including up-regulated proteins $(\operatorname{csp} A, \operatorname{csh} A, h s p 31, m r e B)$ and down-regulated proteins ( $(n a K, f a b D$, groES, fruB). transferase system (PTS) (path: ko02060) were also differently expressed. Further functions of these differently expressed proteins are discussed in the following section.

\section{Transcriptional Expression Analysis of Selected Proteins as Revealed by qRT-PCR}

To confirm the accuracy of the iTRAQ ratios, correlation between some representative proteins with their cognate gene expressions was analyzed. The results were shown as the mean fold changes standard deviation, and significant differences were determined using one-way ANOVA $(p<0.05)$. As shown in Fig. 5, the mRNA levels of cspA, cshA, fanD, groEL, fruB were correlated well with the iTRAQ proteomic results. However, expression of genes hsp31 and mreB was significantly up-regulated, and gene dnaK significantly down-regulated, probably due to complex translational regulations in L. plantarum. The overall trend between the gene and protein expression level was consistent, indicating the credibility of the iTRAQ ratios in the present study.

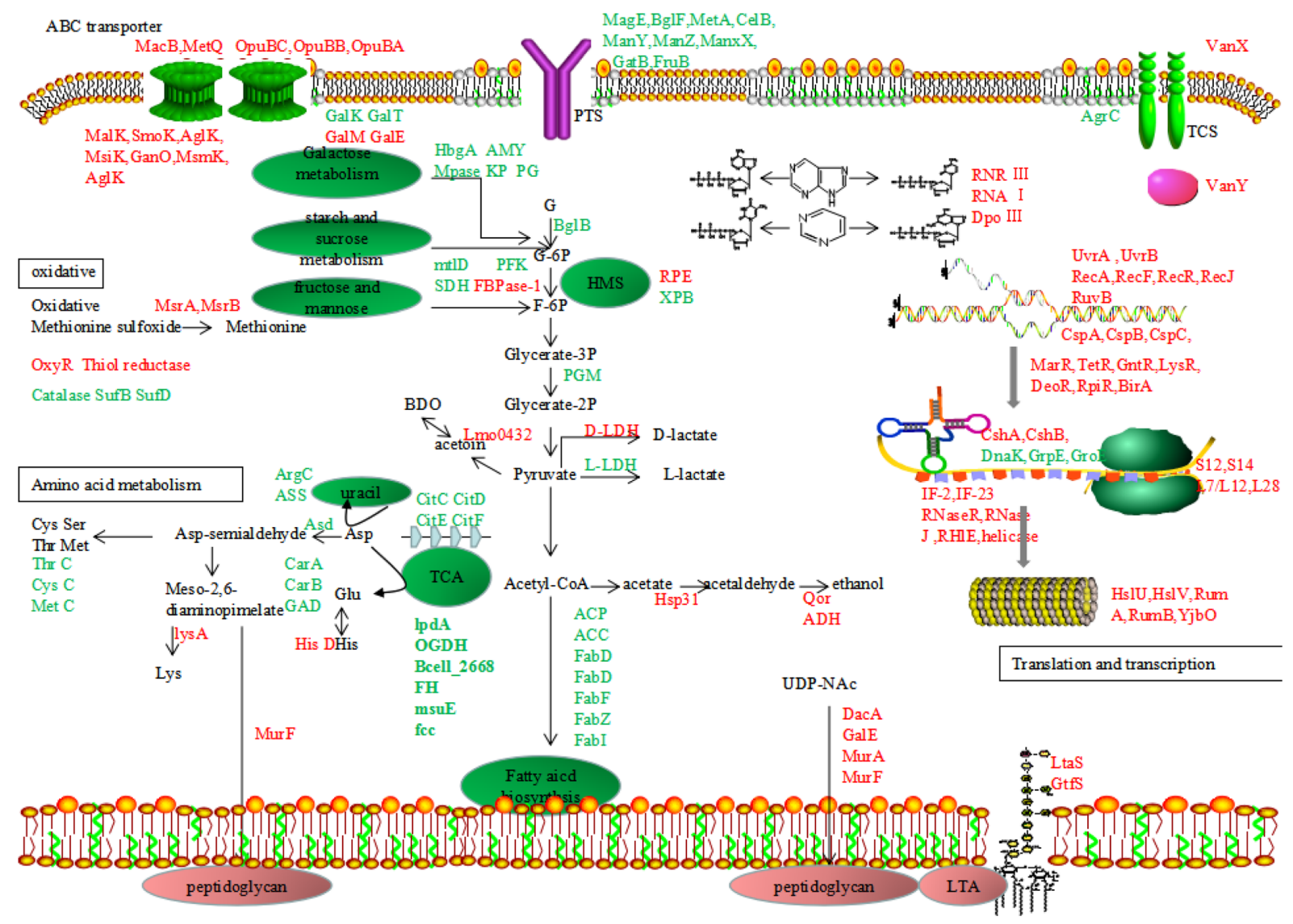

Fig. 5. Proposed model for response mechanisms of L. plantarum K25 under cold stress condition.

Up-regulated and down-regulated proteins (Fold change $>1.5$ or $<0.667, p<0.05$ ) are presented in the color of red and green, respectively. 


\section{Discussions}

Lowering temperature may decrease bacterial growth and proliferation. Meanwhile, the bacteria stimulate selfadjusting to develop cold-resistance mechanisms. The results of the present study revealed that L. plantarum K25 displayed a complex biological network to tackle coldstress mainly by adjusting carbohydrate, amino acid and fatty acid metabolism and biosynthesis. The cold-resistant mechanism of this strain involved a specific energy conservation, survival mode and repairing system, and enhanced protein synthesis ability. Relevant chaperons and transcription regulators against cold stress were also identified.

In this study, we found that $29 \mathrm{DE}$ proteins were involved in carbohydrate metabolism and energy production and conversion including 6 proteins up-regulated and 23 proteins down-regulated, indicating that metabolic nutrient intake and energy production were inhibited to reduce the metabolic level and growth rate at low temperature. These DE proteins participated in glycolysis/glucoenogensis, citrate cycle (TCA), pentose phosphate pathway (PPP), starch and sucrose metabolism, fructose and mannose metabolism and pyruvate metabolism. Down-regulated proteins indicated that during cold-stress condition, bacteria strictly inhibited intake of fructose and mannose which might be needed for bacterial growth. In addition, there was decreased expression of DE proteins involved in sucrose, galactose and cellulose metabolism, which indicated repression of carbohydrate metabolism under low temperature condition. Similar decrease in expression of proteins related to carbohydrate metabolism in $L$. kefiranofaciens M1 and L. sakei in response to cold-stress condition was reported earlier [15, 16].

A large group of proteins with different concentrations was related to amino acid biosynthesis and metabolism. Histidinol dehydrogenase (lp-2172) (responsible for histidine biosynthesis) was upregulated 1.827 -fold. Histidine is an amino acid that has various biological functions such as supplying materials for protein synthesis, being a precursor of physiologically activate substrates and regulating hormone secretion and cell turnover [17]. Besides, we observed down-regulation of the chill-repress protein was involved in the arginine biosynthesis and catalysis of the urea cycle initial step, respectively, using arginine as a substrate for ammonia generation. Down-regulation of carbamoyl phosphate synthase large subunit (lp-2284), carbamoyl phosphate synthase small subunit (lp-2285) (catalysis of the transformation of L-glutamine and carbamoyl phosphate) resulted in accumulation of glutamate and glutamine in cytoplasm which can be used as osmotic protectants to enhance cell survivability and relieve oxidative damage [18]. Up-regulation of diaminopimelate decarboxylase (lp-1432) to increase its cellular abundance promoted transformation of meso-2,6-diaminopimelate into lysine. The intermediates formed in the lysine biosynthesis pathway were also required for peptidoglycan biosynthesis. Secretion of both peptidase C69 (lp-0312, lp-0223) and XaaPro aminopeptidase (lp-1326) increased at $10^{\circ} \mathrm{C}$, suggesting that $L$. plantarum $\mathrm{K} 25$ would scavenge extracellular nitrogen and carbon sources after exposure to an oligotrophic environment [19].

Fatty acid biosynthesis was negatively affected in $L$. plantarum K25 under cold-stress condition. These results indicated that cold stress would cause L. plantarum K25 to reduce membrane fluidity and lead to an inefficient membrane-associated function (e.g. active transport and protein secretion). Besides, down-regulation of proteins involved in fatty acid metabolism also decreased energy production, suggesting a mechanism of energy saving in $L$. plantarum K25 for its survival under cold-stress condition. As the cell wall is the first line of defense against environment stress for bacteria, the enhancement of cell wall component biosynthesis may be a self-protective mechanism of L. plantarum K25 in response to cold. Some proteins were up-regulated to increase total peptidoglycan production. It was noted that peptidoglycan biosynthesis in $S$. aureus cell wall also increased after prolonged cold exposure [20].

In this study, numerous chaperons were identified in $L$. plantarum K25 at low temperature. These proteins could be treated as cellular garbage and undergo proteolysis with aid of specific chaperons [21].The Csp (cold shock protein) family such as CspA, CspB and CspC could respond to cold shock and act as RNA chaperons to improve the protein synthesis capacity by specifically binding to singlestranded nucleic acids, thereby reducing formation of mRNA secondary structure [22]. Therefore, these proteins were essential for effective protein synthesis and cell viability at low temperature.

Structural integrity of DNA molecules is sensitive to changes in the physiological environment. Therefore, the enhancement of protein expression associated with DNA repair, recombination and stabilization in L. plantarum K25 exposed to cold stress indicated that there might be a DNA injury after cell experienced cold stress and an excellent 
DNA repair was needed [23]. Beyond that, 22 DE proteins were identified as transcription factors (TFs), which were involved in numerous cellular functions, including oxidative stress responses, cell wall shape, quorum sensing, efflux pumps, secretion, motility, nitrogen fixation, virulence, cell division, metabolism and environmental recognition. These proteins regulate a wide range of cellular activities including stress response, efflux pump production, and modulation of metabolism [24, 25].

Some proteins increased their abundance upon cold shock, suggesting that these proteins might be involved in inter- or intra-cell signaling system including twocomponent system (TCS), ATP-binding cassette (ABC) transporter and quorum sensing (QS) [26]. Change of gene expression related to TCS and QS relied on external signaling and cell density, respectively [27, 28] Relationship between cold stress and oxidative stress was evidenced in L. monocytogenes at the transcription level [29], but it was rarely studied in lactic acid bacteria. It was known that under cold stress condition, solubility of gasses and production of toxic reactive oxygen species (ROS) increased significantly, thus triggering expression of specific proteins to protect cell machinery from oxidative damage.

In conclusion, the molecular mechanism of the cold-stress response of probiotic L. plantarum K25 was investigated by comparative and functional proteomic analysis. A total of 2,063 proteins were identified, and 506 proteins were found to be differentially expressed. By analyzing the critical DE proteins, L. plantarum K25 was revealed to employ a coldadaptation mechanism mainly by regulating carbohydrate metabolism and energy production, fatty acid biosynthesis, and amino acid biosynthesis, and the proposed molecular mechanism of L. plantarum $\mathrm{K} 25$ in response to cold stress based on the results of this study was diagramed in Fig. 5. Under cold-stress condition at $10^{\circ} \mathrm{C}$, carbohydrate metabolism was inhibited to reduce bacterial growth rate and save energy for survival. Decreased fatty acid biosynthesis and metabolism led to change of membrane composition and reduced cell membrane fluidity, while increased biosynthesis of peptidoglycan and LTA significantly enhanced cell wall integrity to protect cells from environmental injury. Furthermore, improved purine and pyrimidine metabolism provided more substrates for DNA biosynthesis, thus increasing transcription and translation efficiency. There were also many chaperons and transcription factors differentially expressed to assist in precise and efficient synthesis of proteins needed for counteracting cold stress. The present study provided further insight into the basic physiology of L. plantarum responding to cold stress, facilitating development of novel strategies to increase bacterial survivability at low temperature.

\section{Competing Interests}

The authors have no financial conflicts of interest to declare.

\section{Acknowledgements}

This work was supported by the grants from the National Natural Science Foundation of China (No. 31871823; No. 31571857) and Beijing Talent Cultivation Quality Construction - First-class Professional Construction (Municipal Level)Food Science and Engineering (PXM2019_014213_000010).

\section{References}

1. Lynch KM, Coffey A, Arendt EK. 2018. Exopolysaccharide producing lactic acid bacteria: Their techno-functional role and potential application in gluten-free bread products. Food Res. Int. 110: 52-61.

2. Cruz AG, Antunes AEC, Sousa ALOP, Faria JA, Saad SMI. 2009. Ice-cream as a probiotic food carrier. Food Res. Int. 42:1233-1239.

3. Woraprayote W, Malila Y, Sorapukdee S, Swetwiwathana A, Benjakul S, Visessanguan W. 2016. Bacteriocins from lactic acid bacteria and their applications in meat and meat products. Meat Sci. 120: 118-132.

4. Shi H, Chen Z, Kan J. 2016. Progress in research on stress response in Escherichia coli during food processing and storage. Food Sci. 37: 250-257.

5. Cacace G, Mazzeo MF, Sorrentino A, Spada V, Malorni A, Siciliano RA. 2010. Proteomics for the elucidation of cold adaptation mechanisms in Listeria monocytogenes. J. Proteomics. 73: 2021-2030.

6. Carranza P, Grunau A, Schneider T, Hartmann I, Lehner A, Stephan $\mathrm{R}$, et al. 2010. A gel-free quantitative proteomics approach to investigate temperature adaptation of the foodborne pathogen Cronobacter turicensis 3032. Proteomics 10: 3248-3261.

7. Alreshidi MM, Dunstan RH, Macdonald MM, Smith ND, Gottfries J, Roberts TK. 2015. Metabolomic and proteomic responses of Staphylococcus aureus to prolonged cold stress. J. Proteomics 121: 44-55.

8. Fournier M, Aubert C, Dermoun Z, Durand MC, Moinier D, Dolla A. 2006. Response of the anaerobe Desulfovibrio vulgaris Hildenborough to oxidative conditions: proteome and transcript analysis. Biochimie 88: 85-94. 
9. Zhang J, Zhang L, Qiu J, Nian H. 2015. Isobaric tags for relative and absolute quantitation (iTRAQ)-based proteomic analysis of Cryptococcus humicola response to aluminum stress. J. Biosci. Bioeng. 120: 359-363.

10. Bakermans C, Tollaksen SL, Giometti CS, Wilkerson C, Tiedje JM, Thomashow MF. 2007. Proteomic analysis of Psychrobacter cryohalolentis K5 during growth at subzero temperatures. Extremophiles 11: 343-354.

11. Barria CM, Malecki M, Arraiano CM. 2013. Bacterial adaptation to cold. Microbiology 159: 2437-2443.

12. Zhang L, Zhang X, Liu C, Li C, Li S, Li T, et al. 2013 Manufacture of Cheddar cheese using probiotic Lactobacillus plantarum K25 and its cholesterol-lowering effects in a mice model. World J. Microbiol. Biotechnol. 29: 127-135.

13. Wang J, Zhao X, Tian Z, Yang Y, Yang Z. 2015. Characterization of an exopolysaccharide produced by Lactobacillus plantarum YW11 isolated from Tibet Kefir. Carbohydr. Polym. 125: 16-25.

14. Zhang J, Zhao X, Jiang $\mathrm{Y}$, Zhao $\mathrm{W}$, Guo $\mathrm{T}$, Cao $\mathrm{Y}$, et al. 2017. Antioxidant status and gut microbiota change in an aging mouse model as influenced by exopolysaccharide produced by Lactobacillus plantarum YW11 isolated from Tibetan kefir. J. Dairy Sci. 100: 6025-6041.

15. Chen MJ, Tang HY, Chiang ML. 2017. Effects of heat, cold, acid and bile salt adaptations on the stress tolerance and protein expression of kefir-isolated probiotic Lactobacillus kefiranofaciens M1. Food Microbiol. 66: 20-27.

16. Marceau A, Zagorec M, Chaillou S, et al. 2004. Evidence for involvement of at least six proteins in adaptation of Lactobacillus sakei to cold temperatures and addition of NaCl. Appl. Environ. Microbiol. 70: 7260-7268.

17. Marceau A, Zagorec M, Chaillou S, Méra T, ChampomierVergès MC. 2017. Histidine deficiency attenuates cell viability in rat intestinal epithelial cells by apoptosis via mitochondrial dysfunction. J. Nutr. Intermed. Metab. 8: 21-28

18. Guo J, Song X, Zou L, Chen G. 2015. The small and large subunits of carbamoyl-phosphate synthase exhibit diverse contributions to pathogenicity in Xanthomonas citri subsp. citri. J Integr. Agr. 14: 1338-1347.

19. Are VN, Kumar A, Kumar S, Goyal VD, Ghosh B, Bhatnagar D, et al. 2017. Crystal structure and biochemical investigations reveal novel mode of substrate selectivity and illuminate substrate inhibition and allostericity in a subfamily of Xaa-Pro dipeptidases. Biochim. Biophys. Acta Proteins Proteom. 1865: 153-164.

20. Onyango LA, Dunstan RH, Gottfries J, von Eiff C, Roberts TK. 2012. Effect of low temperature on growth and ultrastructure of Staphylococcus spp. PLoS One. 7: e29031.

21. Craig EA, Gambill BD, Nelson RJ. 1993. Heat shock proteins: molecular chaperones of protein biogenesis. Microbiol. Mol. Biol. R. 57: 402-414.

22. Graumann P, Marahiel MA. 1996. Some like it cold: response of microorganisms to cold shock. Arch Microbiol. 166: 293-300.

23. Juliana D, Tom R, Bowman JP. 2013. Characterisation of the transcriptomes of genetically diverse Listeria monocytogenes exposed to hyperosmotic and low temperature conditions reveal global stress-adaptation mechanisms. PLoS One 8: e73603.

24. Deng W, Li C, Xie J. 2013. The underling mechanism of bacterial TetR/AcrR family transcriptional repressors. Cell Signal. 25: 1608-1613.

25. Juan L. Ramos, Manuel Martínez-Bueno, Antonio J. MolinaHenares, Wilson Terán, Kazuya Watanabe, Xiaodong Zhang, et al. 2005. The TetR family of transcriptional repressors. Microbiol. Mol. Biol. Rev. 69: 326-356.

26. Steele KH, O'Connor LH, Burpo N, Kohler K, Johnston JW. 2012. Characterization of a ferrous iron-responsive twocomponent system in nontypeable haemophilus influenzae. J. Bacteriol. 194: 6162-6173.

27. Iwata Y, Satou K, Tsuzuku H, Furuichi K, Senda Y, SakaiTakemori Y, et al. 2017. Down-regulation of the twocomponent system and cell-wall biosynthesis-related genes was associated with the reversion to daptomycin susceptibility in daptomycin non-susceptible methicillinresistant Staphylococcus aureus. Eur. J. Clin. Microbiol. 36: 1839-1845.

28. Miller MB, Bassler BL. 2001. Quorum sensing in bacteria. Annu. Rev. Microbiol. 55: 165-199.

29. Liu S, Graham JE, Bigelow L, Morse PD 2nd, Wilkinson BJ. 2002. Identification of listeria monocytogenes genes expressed in response to growth at low temperature. Appl. Environ. Microbol. 68: 1697-1705. 